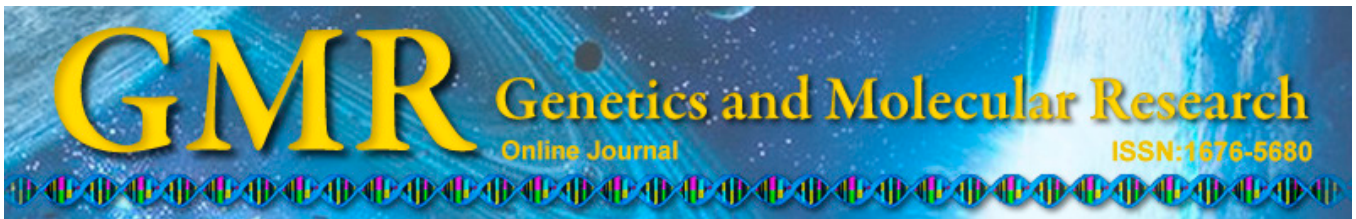

\title{
Genome-wide association study of growth traits in the Jinghai Yellow chicken
}

\author{
G.X. Zhang ${ }^{1 *}$, Q.C. Fan ${ }^{1 *}$, T. Zhang ${ }^{1}$, J.Y. Wang ${ }^{1}$, W.H. Wang ${ }^{1}$, Q. Xue ${ }^{1}$ \\ and Y.J. Wang ${ }^{2}$ \\ ${ }^{1}$ College of Animal Science and Technology, Yangzhou University, \\ Yangzhou, China \\ ${ }^{2}$ Jiangsu Jinghai Industry Poultry Group Co., Ltd., Jiangsu Nantong, China \\ *These authors contributed equally to this study. \\ Corresponding author: J.Y. Wang \\ E-mail: jywang@yzu.edu.cn
}

Genet. Mol. Res. 14 (4): 15331-15338 (2015)

Received June 27, 2015

Accepted October 8, 2015

Published November 30, 2015

DOI http://dx.doi.org/10.4238/2015.November.30.10

\begin{abstract}
Growth is one of the most economically important traits in the poultry industry. In this study, we identified singlenucleotide polymorphisms (SNPs) and candidate genes associated with growth traits of the Jinghai Yellow chicken. Genome-wide association studies were conducted using the Illumina $60 \mathrm{~K}$ SNP Chicken array to genotype 400 Jinghai Yellow chickens. For each bird, the body weights at hatching and at 2, 4, 6, 8, 12, 14, and 16 weeks were recorded. The SNPs that were significantly associated with the growth traits were identified using the general linear regression model. The results revealed a total of 18 SNPs that reached Bonferroni genome-wide significance $(\mathrm{P}<1.80 \mathrm{E}-6)$. Three proximal genes (BTRC, NLK, and NFI) were found to participate in the Wntsignaling pathway and mitogen-activated protein kinase signaling pathway. Haplotype analysis identified 19 significant haplotypes and identified a region 152.4-156.3M on GGA1 affecting 3 growth traits (BW4, BW14, and BW16). These results may help identify the exact
\end{abstract}


locations of body weight quantitative trait loci on a genome level and indicate variants that can be used for subsequent investigations for Jinghai Yellow chicken.

Key words: Body weight; Genome-wide association study; PLINK; Jinghai Yellow chicken; Single-nucleotide polymorphism

\section{INTRODUCTION}

Growth is one of the most economically important traits in the poultry industry; therefore, studies exploring quantitative trait loci (QTLs) that affect the body weight of chickens are very important (Wahlberg et al., 2009; Gu et al., 2011; Xie et al., 2012). To date, more than 1500 QTLs, covering most of the genome, have been found to be associated with growth traits (Hu et al., 2013). Previous genomic studies have generally employed low-density microsatellites as markers. However, this approach may no longer provide novel information.

Currently, genome-wide association studies (GWAS) employ single-nucleotide polymorphisms (SNPs) as markers; these potential markers are distributed throughout the entire genome at a high density. Such studies have been used to comprehensively analyze complex, economically important traits using technical statistical tools (Fan et al., 2011; Liu et al., 2011; Onteru et al., 2012; Liu et al., 2013; Wolc et al., 2014). Thus, GWAS have been one of the most effective approaches for identifying related QTLs and functional genes (Xu et al., 2013). In chickens, Sun et al. (2013) identified 14 new genes related to meat quality traits in chicken using a GWAS. Liu et al. (2013) found that a consistent region on chicken GGA4 was associated with carcass weight and eviscerated weight. Gu et al. (2011) used an $\mathrm{F}_{2}$ resource population derived from Silky Fowl and White Plymouth Rock chickens to detect SNPs associated with body weight at 7-14 weeks of age in a region of GGA4 (71.6-80.2 Mb). Xie et al. (2012) identified SNPs in a region of GGA1 $(173.5-175 \mathrm{Mb})$ that strongly affected the body weight of $\mathrm{F}_{2}$ chickens based on an $\mathrm{F}_{2}$ population derived from Xinghua and White Plymouth Rock chickens.

Chinese chicken breeds have gained increased attention for their high nutritional value and improved meat quality, although most breeds are slow-growing. Jinghai chicken is a national cultivated meat breed (minitype). In order to increase the growth rate of this chicken breed, the Illumina $60 \mathrm{~K}$ SNP Chicken array was used to identify key SNPs and functional genes affecting growth traits in the present study.

\section{MATERIAL AND METHODS}

\section{Ethics statement}

Blood samples of chickens were collected from the brachial vein using a standard venipuncture procedure (\#XK622) approved by the Animal Welfare Committee of Yangzhou University.

\section{Experimental animals}

Jinghai Yellow chickens were obtained from the core group within the Jinghai Yellow 
Chicken Breeding Station. Nineteen unrelated male chickens were selected to constitute 19 half-sibling families, and 400 female offspring were chosen randomly from the same batch. All chickens were raised in the pens and fed commercial diets meeting National Research Council's (NRC) requirements. Their body weights at hatching and at 2, 4, 6, 8, 12, 14, and 16 weeks were recorded. Before GWAS, the data were transformed in Minitab (v16.1.1) to approximate a standard normal distribution.

\section{Sample preparation}

The Dzup Genomic DNA Isolation Reagent Kit from Sangon Biotech Co., Ltd. (Shanghai, China) was used to extract genomic DNA, and a NanoDrop 2000 was used to assay the concentration and quality of genomic DNA to meet the requirements of the Infinium SNP genotyping platform. Subsequently, the DNA samples were genotyped using the Illumina 60 K SNP Chicken array (DNA LandMarks Inc., Quebec, Canada).

\section{Data preparation}

Quality control of the returned data was performed using PLINK (v1.07) (Purcell et al., 2007), and SNPs were removed for failing to meet one or more of the following criteria: low call rate $(<90 \%)$, low call frequency $(<95 \%)$, low Hardy-Weinberg equilibrium $(<1 \mathrm{E}-6)$, and low minor allele frequency $(<3 \%)$. As a result of this process, 396 samples and 46,665 SNPs were retained for further analysis.

Linkage disequilibrium based on SNP pruning of autosomes was applied using the indep-pairwise option with a window size of $25 \mathrm{SNPs}$, a step of $5 \mathrm{SNPs}$, and an $\mathrm{r}^{2}$ threshold of 0.2 , resulting in 12,877 independent SNPs. Principal component analysis (PCA) was performed in GCTA (v1.24), and PCA1 and PCA2 were used in models to reduce the population stratification effect (Wall et al., 2003). Moreover, haplotype analysis was performed with an $\mathrm{r}^{2}$ threshold $(>0.8)$ and window size $(200 \mathrm{~kb})$ in PLINK, and 5650 haplotypes were obtained for further study.

\section{Statistical analysis}

The general linear regression model (GLM, I) in PLINK was used in this study. The model was as follows:

$$
\mathrm{Y}=\mathrm{G} \alpha+\mathrm{X} \beta+\mathrm{e}
$$

where $Y$ is the vector of observations; $G$ is genetic effect vector, $X$ is a matrix containing all other fixed effects, including population structure (PCA1 and PCA2); $\alpha$ and $\beta$ are incidence matrix, and $e$ is the vector of random residual. Furthermore, association analysis between haplotypes and growth traits was also performed using the GLM. The Bonferroni P value was calculated based on the independent SNPs of autosomes, which were defined using the indep-pairwise option with $\mathrm{r}^{2}>0.4$ (Liu et al., 2013). Ultimately, 27,824 SNPs were identified. The threshold P value for Bonferroni potential significance was 3.59E-5 $(1 / 27,824)$, and the $\mathrm{P}$ value for Bonferroni genome-wide significance was $1.80 \mathrm{E}-6(0.05 / 27,824)$. An empirical genome-wide $\mathrm{P}$ value was obtained using the maxT function in PLINK. Manhattan plots were 
created using the R (2.15.1) software.

\section{RESULTS}

Descriptive statistics of the phenotypic measurements of growth traits in the 400 Jinghai Yellow chickens used for the present GWAS studies are shown in Table 1. All non-normal phenotypic data were normalized after Johnson transformation. Manhattan plots of the growth traits with significant SNPs are shown in Figure 1. A total of 18 SNPs with genome-wide significance $(\mathrm{P}<1.80 \mathrm{E}-6)$ were shown (Table 2) to affect BW4 (2), BW6 (1), BW12 (3), BW14 (2), and BW16 (12). Among these SNPs, 6 were located on GGA1 and 4 were located on GGA19. SNPs affecting body weight at 2, 8, and 10 weeks (BW2, BW8, and BW10) were not found.

Table 1. Basic growth-trait statistics.

\begin{tabular}{lcrrrr}
\hline Phenotype $^{1}$ & Sample size & Max & Min & Mean & Standard deviation \\
\hline BW0(g) & 344 & 46 & 25 & 33.9 & 3.7 \\
BW2(g) & 300 & 126 & 40 & 85.6 & 13.5 \\
BW4(g) & 348 & 324 & 106 & 205.3 & 29.9 \\
BW6(g) & 343 & 495 & 200 & 330.2 & 52.1 \\
BW8(g) & 293 & 695 & 310 & 513.5 & 72.7 \\
BW10(g) & 288 & 1290 & 405 & 706.4 & 106.7 \\
BW12(g) & 273 & 1170 & 605 & 870.7 & 100.5 \\
BW14(g) & 335 & 1480 & 680 & 1042.3 & 113.8 \\
BW16(g) & 398 & 1502 & 725 & 1123.5 & 124.8 \\
\hline
\end{tabular}

${ }^{1} \mathrm{BW} 2, \mathrm{BW} 4, \mathrm{BW} 6, \mathrm{BW} 8, \mathrm{BW} 10, \mathrm{BW} 12, \mathrm{BW} 14$, and BW16 indicate the body weights at the ages of 2, 4, 6, 8, 10, 12,14 , and 16 weeks, respectively.

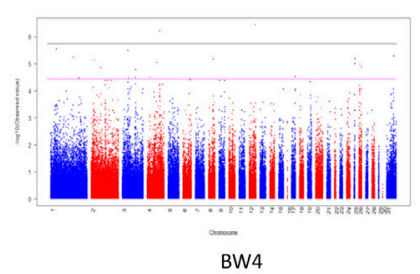

BW4
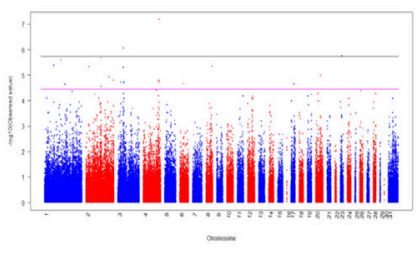

BW12

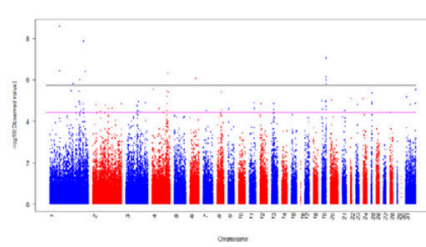

BW16

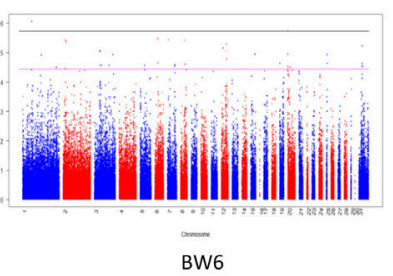

BW6

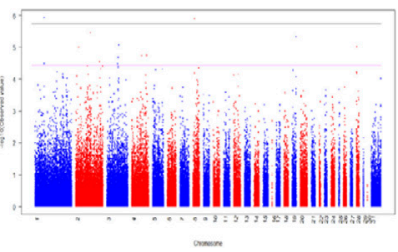

BW14

Figure 1. Manhattan plots for 5 growth traits with genome-wide significant SNPs. 1-28 on the x-axis indicate chromosomes 1-28, and 29, 30, and 31 indicate LGE22, LGE64, and chromosome Z respectively. The magenta horizontal line shows the potential significance threshold:- $\log 10(3.59 \mathrm{E}-5)$, while the black shows the potential significance genome-wise significance threshold:- $\log 10(1.80 \mathrm{E}-06)$. 
Table 2. SNPs that reached genome-wide significance and their proximal genes.

\begin{tabular}{llrrccccl}
\hline Traits & SNP ID & Chr $^{1}$ & \multicolumn{1}{c}{ Pos (bp) } & Alleles $^{2}$ & MAF & Pr $^{3}$ & P-adj & Proximal genes $^{4}$ \\
\hline BW4 & rs14047064 & 12 & 17762137 & AG & 0.03283 & $3.66 \mathrm{E}-07$ & 0.010178 & LOC771741 \\
& GGaluGA263709 & 4 & 68188204 & GA & 0.4633 & $6.05 \mathrm{E}-07$ & 0.016839 & FRYL \\
BW6 & rs13652021 & 1 & 50604748 & CT & 0.08418 & $8.71 \mathrm{E}-07$ & 0.024207 & 22 U LOC101752136 \\
BW12 & GGaluGA267974 & 4 & 85148698 & CT & 0.4508 & $6.57 \mathrm{E}-08$ & 0.001829 & HTT \\
& rs14332284 & 3 & 29814153 & CT & 0.2184 & $8.7 \mathrm{E}-07$ & 0.024193 & 94 U MOCS1 \\
& rs16188810 & 23 & 3101987 & CT & 0.3099 & $1.75 \mathrm{E}-06$ & 0.04872 & PTPRU \\
BW14 & rs13652021 & 1 & 50604748 & CT & 0.08418 & $1.19 \mathrm{E}-06$ & 0.032971 & 22 U LOC101752136 \\
& rs14637110 & 8 & 6077034 & AG & 0.3671 & $1.27 \mathrm{E}-06$ & 0.035336 & CACNA1E \\
BW16 & rs13652021 & 1 & 50604748 & CT & 0.08418 & $2.62 \mathrm{E}-09$ & $7.28 \mathrm{E}-05$ & 22 U LOC101752136 \\
& rs13973774 & 1 & 175116535 & CT & 0.2273 & $1.35 \mathrm{E}-08$ & 0.000375 & 62 U COG6 \\
& rs14123975 & 19 & 9331455 & CT & 0.351 & $8.49 \mathrm{E}-08$ & 0.002361 & SGSM2 \\
& GGaluGA016599 & 1 & 50616709 & AC & 0.06962 & $3.64 \mathrm{E}-07$ & 0.010128 & 10 U LOC101752136 \\
& rs13983925 & 1 & 184074501 & AG & 0.1835 & $3.84 \mathrm{E}-07$ & 0.010682 & 20 U LOC101748238 \\
& GGaluGA267023 & 4 & 82146031 & AG & 0.3987 & $4.68 \mathrm{E}-07$ & 0.013008 & EVC \\
& rs15855551 & 19 & 9232211 & GA & 0.3797 & $7.01 \mathrm{E}-07$ & 0.019496 & NLK \\
& rs14583610 & 6 & 24330708 & TC & 0.1324 & $8.58 \mathrm{E}-07$ & 0.023862 & BTRC \\
& r13957061 & 1 & 154795787 & CT & 0.3023 & $1.00 \mathrm{E}-06$ & 0.027824 & 303 D SLITRK6 \\
& r13576057 & 19 & 9018028 & TG & 0.4356 & $1.03 \mathrm{E}-06$ & 0.028547 & NF1 \\
& r15397087 & 1 & 120766034 & TC & 0.4337 & $1.55 \mathrm{E}-06$ & 0.043099 & 6 U LOC101749456 \\
& rs15050713 & 19 & 9249624 & AC & 0.4293 & $1.57 \mathrm{E}-06$ & 0.043656 & NLK \\
\hline
\end{tabular}

${ }^{1}$ Position based on WADHUC2. ${ }^{2}$ First allele is minor allele. ${ }^{3} \mathrm{Pr}$ indicates P value calculated by GLM, and P-adj indicated $\mathrm{P}$ value corrected by Bonferoni. ${ }^{4} \mathrm{U}=$ upstream, $\mathrm{D}=$ downstream. The unit of distance is $\mathrm{kb}$.

\section{Important candidate genes}

Genes that were closest to the SNPs reaching genome-wide significance were searched in the NCBI database, and 16 genes were identified. Subsequently, pathway analysis of the genes was performed in the KEGG database. Only molybdopterin synthase, betatransducin repeat containing E3 ubiquitin protein ligase (BTRC), nemo-like kinase (NLK), and neurofibromin 1 were found to be involved in pathways of chicken. Furthermore, BTRC and NLK were found to participate in the Wnt pathway: KEGG (gga04310) and NLK and neurofibromin 1 were involved in the mitogen-activated protein kinase (MAPK) pathway: KEGG (gga04010).

\section{Haplotype analysis}

Association analysis was performed between 5650 haplotypes and growth traits, and 19 haplotypes (Table 3) with genome-wide significance were identified to affect BW4 (1), BW6 (2), BW12 (2), BW14 (2), and BW16 (12). Twelve of the 19 haplotypes were located on GGA1 and 7 of the 12 haplotypes were located in the region $52.4-156.3 \mathrm{Mb}$ and affected BW6, $\mathrm{BW} 14$, and BW16, respectively.

\section{DISCUSSION}

Gu et al. (2011) found that SNPs associated with body weight at 7-14 weeks were in a region of GGA4 (71.6-80.2 Mb) and Xie et al. (2012) identified SNPs in a region of GGA1 $(173.5-175 \mathrm{Mb})$ that strongly affected chicken body weight. In the present study, 18 SNPs reaching genome-wide significance in this study were located on 8 different chromosomes. No 
obvious central region was found. Among these SNPs, 6 were located on GGA1, indicating that GGA1 was an important candidate gene affecting body weight traits in the Jinghai Yellow chicken. The most important SNP appeared to be rs13652021, which was located $22 \mathrm{~kb}$ upstream of LOC101752136 and reached genome-wide significance with the BW6, BW14, and BW16 traits.

\begin{tabular}{|c|c|c|c|c|c|c|c|c|c|}
\hline Traits & $\mathrm{Chr}$ & Pos I (bp) $)^{1}$ & SNP I & Pos II (bp) & SNP II & Haplotype & Frequency & $\mathrm{P}^{2}$ & P-adj \\
\hline BW4 & 4 & 68174492 & rs16428332 & 68188204 & GGaluGA263709 & $\mathrm{AA}$ & 0.529 & $2.74 \mathrm{E}-07$ & $7.62 \mathrm{E}-03$ \\
\hline BW6 & 1 & 106629958 & rs14866838 & 106815298 & rs 14866901 & GCGGG & 0.0139 & $3.05 \mathrm{E}-07$ & $8.49 \mathrm{E}-03$ \\
\hline BW6 & 1 & 154795787 & rs13957061 & 154802557 & rs13957071 & CG & 0.199 & 4.99E-07 & $1.39 \mathrm{E}-02$ \\
\hline BW12 & 3 & 29814153 & rs14332284 & 29832007 & rs 14332342 & $\mathrm{CC}$ & 0.216 & $1.56 \mathrm{E}-06$ & $4.34 \mathrm{E}-02$ \\
\hline BW12 & 24 & 2659651 & GGaluGA191711 & 2668989 & GGaluGA191716 & AT & 0.845 & $1.48 \mathrm{E}-06$ & $4.12 \mathrm{E}-02$ \\
\hline BW14 & 1 & 152462032 & rs15461150 & 152587110 & rs 13956096 & GGGG & 0.0973 & $5.89 \mathrm{E}-07$ & $1.64 \mathrm{E}-02$ \\
\hline BW14 & 1 & 173531349 & rs14916724 & 173536168 & GGaluGA054842 & $\mathrm{AG}$ & 0.17 & $1.63 \mathrm{E}-07$ & $4.54 \mathrm{E}-03$ \\
\hline BW16 & 1 & 50616709 & GGaluGA016599 & 50632934 & rs 13863600 & $\mathrm{AG}$ & 0.0696 & $3.18 \mathrm{E}-07$ & $8.85 \mathrm{E}-03$ \\
\hline BW16 & 1 & 120766034 & rs15397087 & 120783594 & rs 13685727 & CT & 0.566 & $1.47 \mathrm{E}-06$ & 4.09E-02 \\
\hline BW16 & 1 & 152462032 & rs15461150 & 152587110 & rs 13956096 & GGGG & 0.0973 & $1.13 \mathrm{E}-06$ & $3.14 \mathrm{E}-02$ \\
\hline BW16 & 1 & 152930210 & rs15461616 & 153065631 & rs 15461695 & TGTCC & 0.243 & $1.70 \mathrm{E}-06$ & 4.73E-02 \\
\hline BW16 & 1 & 154795787 & rs13957061 & 154802557 & rs 13957071 & CG & 0.199 & $1.61 \mathrm{E}-07$ & $4.48 \mathrm{E}-03$ \\
\hline BW16 & 1 & 154795787 & rs13957061 & 154802557 & rs13957071 & TG & 0.698 & $2.60 \mathrm{E}-07$ & $7.23 \mathrm{E}-03$ \\
\hline BW16 & 1 & 156277771 & rs 15465132 & 156390993 & GGaluGA050635 & ATGCGG & 0.072 & $5.21 \mathrm{E}-07$ & $1.45 \mathrm{E}-02$ \\
\hline BW16 & 1 & 173531349 & rs14916724 & 173536168 & GGaluGA054842 & $\mathrm{AG}$ & 0.17 & 4.31E-09 & $1.20 \mathrm{E}-04$ \\
\hline BW16 & 25 & 2007976 & GGaluGA194718 & 2016848 & GGaluGA194725 & ACT & 0.257 & $4.82 \mathrm{E}-07$ & $1.34 \mathrm{E}-02$ \\
\hline BW16 & 25 & 2007976 & GGaluGA194718 & 2016848 & GGaluGA194725 & $\mathrm{ACC}$ & 0.574 & 7.61E-07 & $2.12 \mathrm{E}-02$ \\
\hline BW16 & $\mathrm{Z}$ & 44593823 & rs14717488 & 44724842 & rs14015587 & GGACC & 0.0606 & $3.80 \mathrm{E}-07$ & $1.06 \mathrm{E}-02$ \\
\hline BW16 & Z & 44743299 & rs14955891 & 44760082 & rs 14015602 & $\mathrm{AC}$ & 0.0601 & 8.95E-08 & $2.49 \mathrm{E}-03$ \\
\hline
\end{tabular}

${ }^{1}$ Pos I and SNP I indicate the position and the SNP ID of the first allele of a haplotype, Pos II and SNP II indicate the position and the SNP ID of the last allele of a haplotype. ${ }^{2} \mathrm{P}$ indicates P value calculated by GLM, and P-adj indicated $\mathrm{P}$ value corrected by Bonferoni.

Sixteen candidate genes were detected in this study, with some genes related to tumors and growth. Most of these genes have not been previously reported in chickens. Among the SNPs in these genes, the largest effects were exerted by rs14047064 in LOC771741 on BW4; rs13652021 $22 \mathrm{~kb}$ upstream of LOC101752136 on BW6, BW14, and BW16; GGaluGA267974 in huntingtin on BW12. The functions of LOC771741 and LOC101752136 remain unclear, although all may encode proteins. Huntingtin encodes a protein termed huntingtin. Wild-type huntingtin was reported to protect neurons and regulate cell apoptosis by binding with p21 protein (Cdc42/Rac)-activated kinase 2 (Leavitt et al., 2006; Luo and Rubinsztein, 2009).

The WNT pathway and the MAPK pathway were identified as being associated with growth traits involving BTRC, NLK, and neurofibromin 1. The Wnt pathway, which has been highly conserved throughout evolution, plays an important role in many biological processes, such as growth, development, and metabolism. Furthermore, this pathway is involved in regulating stem cell function in adult tissues, the development of muscle fibers, and the number of terminally myogenic cells (Anakwe et al., 2003; Borello et al., 2006). The MAPK pathway, which is also highly conserved between different species, can participate in many cell processes, such as cell growth, differentiation, migration, and apoptosis (Roberts et al., 2000). BTRC encodes a member of the F-box protein family that functions in phosphorylationdependent ubiquitination. BTRC was found to be a tumor-inhibiting factor in previous studies (Wolter et al., 2003). $N L K$ is involved in both the Wnt pathway and the MAPK pathway. Previous reports indicated that NLK inhibited Wnt/ $\beta$-catenin and regulated cell growth and 
apoptosis in Drosophila and mice (Kortenjann et al., 2001). Pathway analysis was limited because the functions of many of these genes are not definitively known (e.g., LOC101752136). However, based on our results, growth is clearly a highly complex biological process that is regulated by multiple pathways.

Nineteen haplotypes reaching genome-wide significance with growth traits were identified, and 7 of the 19 haplotypes contained SNPs with genome-wide significance. The results not only validated the results of our SNP association study, but also demonstrate that haplotype analysis can be used to identify QTLs that were lost by SNP analysis. Seven of the 19 haplotypes were located in the region 152.4-156.3 Mb of GGA1 and were associated with affected BW6, BW14, and BW16 traits. This region should be further examined. Few studies have examined genes in this region, and only a few of functional genes were identified. These genes mainly found to belong to the SLITRK family (SLITRK1, SLITRK5, and SLITRK6) and are expressed mainly in the central nervous system and hematopoietic stem cells in human and mouse (Milde et al., 2007; Beaubien and Cloutier, 2009).

\section{CONCLUSIONS}

Eighteen SNPs with genome-wide significance were identified to affect growth traits in the Jinghai Yellow chicken. A few candidate genes that affected growth traits were also detected, and 2 possible pathways were found to regulate the growth of Jinghai Yellow chicken. Haplotype analysis identified 19 significant haplotypes and a region 151.3-156.7 Mb on GGA1 that affected 3 growth traits. These results may be useful for identifying the exact locations of body weight QTL on a genome level and provide suggested variants for further studies in Jinghai Yellow chicken.

\section{Conflicts of interest}

The authors declare no conflict of interest.

\section{ACKNOWLEDGMENTS}

Research supported by the National Broiler Industrial and Technology System (\#nycytx-42-G1-05), the Priority Academic Program Development of Jiangsu Higher Education Institutions, and the New Century Talent Project of Yangzhou University.

\section{REFERENCES}

Anakwe K, Robson L, Hadley J, Buxton P, et al. (2003). Wnt signalling regulates myogenic differentiation in the developing avian wing. Development 130: 3503-3514.

Beaubien F and Cloutier JF (2009). Differential expression of Slitrk family members in the mouse nervous system. Dev. Dyn. 238: 3285-3296.

Borello U, Berarducci B, Murphy P, Bajard L, et al. (2006). The Wnt/b-catenin pathway regulates Gli-mediated Myf5 expression during somitogenesis. Development 133: 3723-3732.

Fan B, Onteru SK, Du ZQ, Garrick DJ, et al. (2011). Genome-wide association study identifies loci for body composition and structural soundness traits in pigs. Plos One 6: e14726.

Gu X, Feng C, Ma L, Song C, et al. (2011). Genome-wide association study of body weight in chicken $\mathrm{F}_{2}$ resource population. Plos One 6: e21872.

Hu ZL, Park CA, Wu XL and Reecy JM (2013). Animal QTLdb: an improved database tool for livestock animal QTL/ 
association data dissemination in the post-genome era. Nucleic Acids Res. 41: D871-D879.

Kortenjann M, Nehls M, Smith AJ, Carsetti R, et al. (2001). Abnormal bone marrow stroma in mice deficient for nemolike kinase, Nlk. Eur. J. Immunol. 31: 3580-3587.

Leavitt BR, Raamsdonk JM, Shehadeh J, Fernandes H, et al. (2006). Wild-type huntingtin protects neurons from excitotoxicity. J. Neurochem. 96: 1121-1129.

Liu R, Sun Y, Zhao G, Wang F, et al. (2013). Genome-wide association study identifies loci and candidate genes for body composition and meat quality traits in Beijing-You Chickens. Plos One 8: e61172.

Liu W, Li D, Liu J, Chen S, et al. (2011). A genome-wide SNP scan reveals novel loci for egg production and quality traits in white leghorn and brown-egg dwarf layers. Plos One 6: e28600.

Luo S and Rubinsztein DC (2009). Huntingtin promotes cell survival by preventing Pak2 cleavage. J. Cell Sci. 122: 875885.

Milde T, Shmelkov S, Jensen K, Zlotchenko G, et al. (2007). A novel family of slitrk genes is expressed on hematopoietic stem cells and leukemias. Leukemia 21: 824-827.

Onteru S, Fan B, Du ZQ, Garrick D, et al. (2012). A whole-genome association study for pig reproductive traits. Anim. Genet. 43: 18-26.

Purcell S, Neale B, Todd-Brown K, Thomas L, et al. (2007). PLINK: a tool set for whole-genome association and population-based linkage analyses. The Am. J. Hum. Genet. 81: 559-575.

Roberts CJ, Nelson B, Marton MJ, Stoughton R, et al. (2000). Signaling and circuitry of multiple MAPK pathways revealed by a matrix of global gene expression profiles. Science 287: 873-880.

Wahlberg P, Carlborg Ö, Foglio M, Tordoir X, et al. (2009). Genetic analysis of an F2 intercross between two chicken lines divergently selected for body-weight. BMC Genomics 10: 248.

Wall ME, Reshtsteiner A and Rocha LM (2003). Singular value decomposition component analysis. In: A practical approach to microarray date analysis. (Berrar DP, Dubitzky W, Granzow M, eds.). Kluwer, Norwell.

Wolc A, Arango J, Jankowski T, Dunn I, et al. (2014). Genome-wide association study for egg production and quality in layer chickens. J. Anim. Breed. Genet. 31: 173-182.

Wolter M, Scharwächter C, Reifenberger J, Koch A, et al. (2003). Absence of detectable alterations in the putative tumor suppressor gene BTRC in cerebellar medulloblastomas and cutaneous basal cell carcinomas. Acta Neuropathol. 106: 287-290.

Xie L, Luo C, Zhang C, Zhang R, et al. (2012). Genome-wide association study identified a narrow chromosome 1 region associated with chicken growth traits. Plos One 7: e30910.

Xu Z, Nie Q and Zhang X (2013). Overview of genomic insights into chicken growth traits based on genome-wide association study and microRNA regulation. Curr. Genomics 14: 137-146. 